\title{
Review
}

\section{Journeys in The Country of The Blind: Entanglement Theory and The Effects of Blinding on Trials of Homeopathy and Homeopathic Provings}

\author{
Lionel R. Milgrom
}

Department of Chemistry, Imperial College, London SW7 2AZ, UK

\begin{abstract}
The idea of quantum entanglement is borrowed from physics and developed into an algebraic argument to explain how double-blinding randomized controlled trials could lead to failure to provide unequivocal evidence for the efficacy of homeopathy, and inability to distinguish proving and placebo groups in homeopathic pathogenic trials. By analogy with the famous double-slit experiment of quantum physics, and more modern notions of quantum information processing, these failings are understood as blinding causing information loss resulting from a kind of quantum superposition between the remedy and placebo.
\end{abstract}

Keywords: quantum entanglement - homeopathy - double-blind randomised controlled trials homeopathic provings - double-slit experiment

\section{Introduction}

History records that at the Battle of Copenhagen in 1801 the then Vice-Admiral Nelson famously chose to ignore a direct order from his Commander-in-Chief to withdraw. Putting a telescope to his blind eye, Nelson said, 'I have only one eye-I have a right to be blind sometimes. I really do not see the signal' (1).

A cavalier piece of battle-winning bravado perhaps, but similar 'signal eradicating' blindness (imposed this time in the name of scientific rigour) (2) could be affecting randomized placebo-controlled trials of (i) homeopathy and (ii) homeopathic pathogenic trials (HPTs) (provings). In case (i), doubleblinding could ensure double-blind randomized controlled trials (DBRCTs) are intrinsically incapable of providing unequivocal evidence for or against this controversial therapy's efficacy beyond placebo; while in the second case (ii), double-blinding could prevent verum and placebo proving groups being distinguished from each other. In this article, we shall examine how these effects of blinding can be rationalized and unified in terms of the latest quantum theoretical notions of macroentanglement (3) and information loss (4).

For reprints and all correspondence: Lionel R. Milgrom,

Department of Chemistry, Imperial College, London SW7 2AZ, UK.

E-mail: 1.milgrom@imperial.ac.uk

\section{RCTs and Ideology: 'If Gold Rust, What Shall Iron Do?'}

Developed as a 'gold standard' for testing efficacy of drugs and therapeutic procedures (5), the reputation of the DBRCT has become somewhat tarnished of late. Reporting in The Lancet, a recent Swiss meta-analysis (6) claimed to show that the efficacy of homeopathy was no better than placebo. This led The Lancet's editor and other commentators, including some elements of the media, to 'read homeopathy its last rights' (7). However, detailed examination of The Lancet meta-analysis shows that out of the well over one hundred trials and previous meta-analyses, the authors actually made use of the only eight that happened to show homeopathy was no better than placebo. Not surprisingly, such faulty methodology, biased conclusions and poor presentation were roundly criticized by many serious researchers in the field (8-12). However, these short-comings have so far failed to make any impression on an essentially biased conventional scientific community, and an equally jaundiced media apparently less intent on objective reporting than writing off homeopathy as dead and buried. It is interesting to speculate why.

The increasing hegemony of evidence-based, biomolecular medicine (13) ensures the continuation of attacks on homeopathy and many CAMs for being at best a placebo response, 
at worst quackery. This is because many of the remedies prescribed by homeopaths are so dilute, no molecules of the original substance can remain. Therefore, homeopathy's detractors argue, homeopathy cannot possibly work because how can nothing do something, i.e. bring about cure? This has led to homeopathy arguably being more severely tested via DBRCTs than any other therapeutic modality (including conventional biomedicine). Although on balance, the number of trials performed seems to favor homeopathy (14-17), skeptics remain unconvinced and repeatedly call for yet more 'definitive' trials that 'prove' homeopathy is no better than placebo (5).

The ideological basis of DBRCTs implicitly assumes that specific effects (e.g. of the remedial substance) and nonspecific (contextual) effects of the therapeutic process (e.g. consultation) can be separated and treated independently of each other (18). Though the DBRCT ethos is fully consistent with a centuries-old reductionist agenda (and its ontological division of observers from the objects of their observation), it is a worldview that has been effectively challenged by the discoveries of modern quantum theory, i.e. non-locality and entanglement.

\section{An Entanglement Metaphor for Homeopathy?}

Entangled entities behave as one inseparable holistic unit, whose totality cannot be deduced from any of its parts. Nonlocality has been defined as 'the mysterious ability of nature to enforce correlations between separated but entangled parts of a quantum system that are out of speed-of-light contact: to reach instantaneously across vast spatial distances, or even across time itself, to ensure that the parts of a quantum system are made to match' (19). This means that observation of one part of an entangled system instantaneously provides information about the rest, provided one knows how the system is entangled. However, attempts to observe the parts of an entangled system as separate entities, destroys the whole.

While non-locality and entanglement have been experimentally demonstrated at the nanoscopic level of particles, atoms and even molecules (20), macroentanglement between everyday objects and people is still a matter of conjecture. Indeed, the non-commuting algebraic formulation of orthodox quantum theory (which contains the extremely small number called Planck's constant, $h=6.626 * 10^{-34} \mathrm{~J} \mathrm{~s}^{-1}$ ) would appear to preclude such quantum effects between macroscopic objects/ entities. Nevertheless, it is proving increasingly possible to conceive non-locality and entanglement in a similar but less restricting algebraic $(21,22)$ or, indeed non-algebraic context (23), and usefully apply these concepts to phenomenological problems arising out of CAM research. Thus, Gernert (24) defines a 'common pre-arranged context' which he suggests characterizes the preparation to be made in advance (or naturally given conditions) in order to enable entanglement. His development of this idea also leads to ways of considering macroentanglement as a possible explanation of significant correlations found between subjects' brain function in carefully performed experiments on spatially separated pairs of human subjects $(25,26)$.

Weatherley-Jones et al. (18) had already suggested the reason DBRCTs apparently fail to unequivocally demonstrate the efficacy of homeopathy, is because specific and non-specific effects of the therapeutic process are actually interdependent and mutually correlated. Thus, according to a holistic paradigm, the methodologies used in DBRCTs, and the loss of information such techniques imply, must necessarily destroy the very thing they are trying to investigate. This theme was developed further into a critique of DBRCT methodology (27) couched in terms of a developing algebraic metaphor of the homeopathic therapeutic process called PPR entanglement (22). The latter considers that macroentanglement between the patient, practitioner and remedy can facilitate the therapeutic process.

Thus, patient $(\mathrm{Px})$, practitioner $(\mathrm{Pr})$, and remedy $(\mathrm{Rx})$ states are envisaged as expressible in terms of wave functions; $\psi_{\mathbf{P x}}$, $\psi_{\mathbf{P r}}, \psi_{\mathbf{R x}}$, each expressing a multitude of states. However, only two for each will be considered. If $|\mathbf{n}\rangle$ denotes a state with wave function $\psi_{\mathbf{n}}$, then in any potentially therapeutic situation, Px may be considered in a state of wellness $(|\mathbf{P x} \uparrow\rangle)$ or unwellness $(|\mathbf{P x} \downarrow\rangle)$; Pr may be helpful $(|\mathbf{P r} \uparrow\rangle)$ or unhelpful $(|\operatorname{Pr} \downarrow\rangle)$; and the remedy may be curative $(|\mathbf{R x} \uparrow\rangle)$ or noncurative $(|\mathbf{R x} \downarrow\rangle)$. Using the three-way entanglement formalism of Greenberger, Zeilinger and Horne (28), a maximally entangled state wave function between Px, Pr and Rx may be written as follows:

$$
\left.\left|\Psi_{\text {PPR }}\right\rangle=\frac{1}{\sqrt{2}}(|\mathbf{P x} \uparrow \operatorname{Pr} \uparrow \mathbf{R x} \uparrow\rangle+\operatorname{Px} \downarrow \operatorname{Pr} \downarrow \mathbf{R x} \downarrow\rangle\right)
$$

Also, in orthodox quantum theory, experimental observations are described by operators. There is a connection between wave functions $(|\psi\rangle$ and its complex conjugate $\langle\psi|)$, operators $(\boldsymbol{\Omega}$, and the observations associated with them), and the outcomes of measurements, leading to an 'expectation value' $(\langle\Omega\rangle)$. This is the mean value of an observable after a series of experimental observations:

$$
\langle\psi|\Omega| \psi\rangle=\langle(\Omega)\rangle
$$

A similar equation can be written to describe the outcome of the therapeutic entangled interaction between $\mathrm{Px}, \mathrm{Pr}$ and $\mathrm{Rx}$ leading to an observed overall change in symptoms, $\boldsymbol{\Delta} \boldsymbol{S} \boldsymbol{x}$ (29):

$$
\left\langle\Psi_{\text {PPR }}|\Pi r| \Psi_{\text {PPR }}\right\rangle=\langle(\boldsymbol{S} \boldsymbol{S x})\rangle
$$

where $\left|\Psi_{\text {PPR }}\right\rangle$ represents the PPR entangled state wave function, $\left\langle\boldsymbol{\Psi}_{\mathbf{P P R}}\right|$ its complex conjugate and $\boldsymbol{\Pi r}$ denotes the 'homeopathic operator' (and the 'therapeutic state space' created by $\mathrm{Pr}$ ).

It is worth noting that although Equations (2) and (3) look superficially similar, in (2) the self-adjoint operator $\boldsymbol{\Omega}$ is an entity essentially independent of that which it operates upon (i.e. the wave function $|\psi\rangle$ and its complex conjugate $\langle\psi|$ ). However, in (3), the practitioner $\mathrm{Pr}$ is functioning both as the 
homeopathic operator $\Pi \mathbf{r}$ and as part of the PPR entangled state wave function $\left|\Psi_{\mathbf{P P R}}\right\rangle$ (and its complex conjugate, $\left.\left\langle\Psi_{\text {PPR }}\right|\right)$ that $\Pi \mathbf{r}$ operates upon. In this sense, the Pr operates to 'reflect back' the state of Px and the notion of cure. Thus the homeopathic operator $\boldsymbol{\Pi r}$ is an 'active mirror'. Pr embodies the homeopathic operator $\Pi \mathbf{r}$, which includes 'generating' the 'state space' in which therapy takes place. In this respect, Equation (3) may be considered non-linear: Pr may be thought of as helping to create both the conditions for cure (the homeopathic operator, $\Pi \mathbf{r}$ ) and being entangled with the curative PPR state. Thus, Pr's function is in helping to create a healing 'space' (also denoted by $\Pi \mathbf{r}$ ) for Px and then appearing and operating within that space as part of the PPR entangled state.

\section{Entanglement-Breaking Effect of DBRCTs on Homeopathy}

Weatherley-Jones et al. (18) describe how two types of DBRCT have been used to test the efficacy of homeopathy in (i) specific, non-individualized homeopathic medicines (3032) and (ii) individualized homeopathic prescribing (33-36).

Generally, DBRCTs deemed of higher quality tend to show less significant results than those of lesser quality. WeatherleyJones et al (18) then ask whether this is '. . . because homeopathy is nothing more than a placebo effect or does the evaluation of a homeopathic approach create particular challenges for a placebo-controlled trial?' The entanglement model developed earlier and outlined in Equations (1) and (3) could help to throw some light upon this question.

\section{DBRCTs of Specific Non-Individualized Homeopathic Medicines}

If a non-individualized homeopathic medicine is considered as one prescribed with little or no intervention by a practitioner, Pr, then in terms of Equations (1) and (3), this would mean there would be no opportunity for entanglement with Pr. Thus, in Equation (1), $|\mathbf{P r}\rangle$ becomes 0. Consequently, Equation (1) reduces to:

$$
\left|\Psi_{\mathbf{P P R}}\right\rangle=\frac{1}{\sqrt{2}}(|\mathbf{P x} \uparrow . \mathbf{0 . R x} \uparrow\rangle+|\mathbf{P x} \downarrow . \mathbf{0 . R x} \downarrow\rangle)=0
$$

In other words, the PPR entangled state wave function (and therefore also that of its complex conjugate $\left.\left\langle\Psi_{\mathbf{P P R}}\right|\right)$ collapse to zero. But a further conclusion can be drawn. With no practitioner $\mathrm{Pr}$, then there can be no homeopathic operator/ state-space, $\boldsymbol{\Pi r}$, i.e. $\boldsymbol{\Pi r}=0$. Substituting into Equation (3) gives:

$$
\left\langle\boldsymbol{\Psi}_{\mathbf{P P R}}|\boldsymbol{\Pi r}| \boldsymbol{\Psi}_{\mathbf{P P R}}\right\rangle=\langle 0|0| 0\rangle=\langle(\boldsymbol{\Delta} \mathbf{S x})\rangle=0
$$

That is, the expectation value $\langle(\boldsymbol{S} \boldsymbol{x})\rangle$ also becomes 0 , which means that no change in symptoms can be expected. Thus, the more rigorously DBRCT methodology is applied to the testing of non-individualized homeopathic medicines, the more an entanglement model of the homeopathic process predicts how they are unlikely to provide significant results in terms of an observable and therapeutic change in symptoms.

\section{DBRCTs of Individualized Homeopathic Prescribing}

The entanglement argument here is more subtle. Clearly, a practitioner $\mathrm{Pr}$ is now involved and may entangle with $\mathrm{Px}$ and Rx, so that initially, Equation (1) holds, i.e.

$$
\left.\left|\Psi_{\text {PPR }}\right\rangle=\frac{1}{\sqrt{2}}(|\operatorname{Px} \uparrow \operatorname{Pr} \uparrow \mathbf{R x} \uparrow\rangle+\operatorname{Px} \downarrow \operatorname{Pr} \downarrow \mathbf{R x} \downarrow\rangle\right)
$$

However, under the conditions of the DBRCT protocol, crucially Pr does not know if the prescribed remedy is verum or placebo.

It is worth pointing out that there are situations in real homeopathic practice where a practitioner might intentionally give a placebo (e.g. sac. lac.) as a second prescription, for example, when Pr has assessed that a previous remedy's action is not exhausted, yet Px is still experiencing symptoms (37). It should be emphasized that this is not the case in an DBRCT designed to test individualized homeopathic prescribing, as although it is the Pr's intention is to give the prescribed remedy, blinding ensures that it remains uncertain as to whether that intention has been met.

How might this affect the therapeutic outcome? 'The homeopaths had been instructed to conduct their consultations as usual and consider that all patients received real homeopathic treatment, not to think about whether patients were on placebo or real treatment, and to consider that lack of reaction to remedies was due to factors documented in the homeopathic literature. Thus, the reaction of patients to any specific effects of the prescription could affect the homeopath, potentially influencing the nature of the consultation. In this way, the specific effect may impact on the non-specific effect' (18).

Thus, in order to comply with implicit assumptions inherent in the DBRCT methodology, homeopathic practitioners are expected to engage in a highly questionable (and ultimately confusing) form of self-deception that would be utterly unthinkable in a real therapeutic situation $(38,39)$.

Thus, in the light of Pr's crucial uncertainty over whether Px received verum or placebo $\Pi \mathbf{r}$ cannot 'reflect' the entangled state wave function $\left|\Psi_{\mathbf{P P R}}\right\rangle$ to give its complex conjugate, $\left\langle\boldsymbol{\Psi}_{\mathbf{P P R}}\right|$, so that $\boldsymbol{\Pi r}=0$, and $\left\langle\boldsymbol{\Psi}_{\mathbf{P P R}}\right|=0$, leading algebraically as follows:

$$
\left\langle\boldsymbol{\Psi}_{\mathbf{P P R}}|\boldsymbol{\Pi r}| \boldsymbol{\Psi}_{\mathbf{P P R}}\right\rangle=\left\langle 0|0| \boldsymbol{\Psi}_{\mathbf{P P R}}\right\rangle=0=\langle(\boldsymbol{\Delta} \boldsymbol{S} \boldsymbol{x})\rangle
$$

That is, same result as in Equation (5), and the expectation value $\langle(\boldsymbol{\Delta} \boldsymbol{S} \boldsymbol{x})\rangle$ tends to 0 , meaning that no change in symptoms can be expected. Thus, the more rigorously individualized homeopathic prescribing is tested via DBRCTs, the more an entanglement model of the homeopathic process predicts they would fail to provide significant results in terms of an observable and therapeutic change in symptoms. 


\section{The DBRCT as an Act of Observation: 'Collapse' of the Therapeutic Effect}

On the basis of these conclusions, it is interesting to consider the DBRCT methodology as an observational process. Equations (5) and (6) indicate that a possible effect of the DBRCT methodology is to reduce the PPR entangled state wave function $\left(\left|\boldsymbol{\Psi}_{\mathbf{P P R}}\right\rangle\right)$ and its complex conjugate $\left(\left\langle\boldsymbol{\Psi}_{\mathbf{P P R}}\right|\right)$, to zero.

In orthodox quantum theory, based on the positivist Copenhagen Interpretation (CI) (40) such a process is deemed to occur as a direct result of the observational process. It is called 'the collapse of the wave function'. While unobserved, a particle is considered to exist in an indeterminate state whose evolution in time is expressed by its wave function. However, observation causes the wave function to 'collapse', and a particle is observed, whose complementary position and momentum are related via Heisenberg's Uncertainty Principle. Thus, in CI, the act of observation in part creates that which is observed. The price of knowledge therefore is the loss of an underlying (ontological) physical reality. It could be argued that by its very nature, the DBRCT is an observational procedure which can 'collapse' $\left|\boldsymbol{\Psi}_{\mathbf{P P R}}\right\rangle$ and its complex conjugate $\left\langle\boldsymbol{\Psi}_{\mathbf{P P R}}\right|$, and is having a similar 'reality-losing' effect here, i.e. the loss or 'collapse' of the homeopathic effect. Thus the PPR entanglement model is implying that the observational stance taken during the DBRCT methodology to the investigation of homeopathy's efficacy essentially disrupts the very thing it is trying to observe. This suggests that the application of the DBRCT methodology to the study of homeopathy is fundamentally flawed, in agreement with Weatherley-Jones et al.'s (18) conclusions based on clinical findings.

There is one further conclusion to be drawn from the above treatment. The loss of information arising out of the effect of blinding to what is remedy and what is placebo, could result in quantum interference, i.e. entanglement, between them. It is therefore the formation of a remedy-placebo entangled state that leads to the breaking of entanglement between patient, practitioner and remedy.

Thus, an algebraic treatment based on a patientpractitioner-remedy macroentanglement theory demonstrates that imposition of blinded DBRCT methodological conditions could effectively proceed as follows:

- Impede PPR entanglement in trials of non-individualized homeopathic remedies (where the remedy has been selected purely on the basis that it has previously been used to treat the conventional medically diagnosed condition, and not on standard homeopathic practice of individualized case taking).

- Break PPR entanglement once it has formed in trials of individualized homeopathic prescribing (where the remedy is selected according to standard homeopathic practice of taking the individual case, but neither test subject nor practitioner know if verum or placebo were actually given during the trial).
The more rigorously, therefore, the blinded DBRCT methodology is applied to testing the efficacy of homeopathy, the more PPR entanglement is reduced (and remedy-placebo entanglement increased) and the less a positive DBRCT outcome is likely. It is true, however, that DBRCTs of homeopathy do sometimes return (albeit small) positive results. The standard response to this by those skeptical of homeopathy's efficacy is to suggest that the blinding in these trials is not perfect (5). Another possibility is that these trials represent some form of statistical fluctuation. In which case, it is perhaps time that other statistical techniques $(39,41)$ were more commonly employed in clinical trials procedures (40). In addition, alternative explanations have been advanced (27) in terms of some surviving residual entanglement. For example, the homeopathic remedy may be thought to entail the entangled intention of those involved in its preparation $(43,44)$ without overarching patient-practitioner-remedy entanglement to 'lock' the remedy into 'therapeutic coherence', this residual entanglement might be enough to deliver the relatively small clinical effect sizes sometimes observed in DBRCTs of homeopathy. In another explanation, residual entanglement may be thought of as surviving at the molecular level from the production of the potentized remedy, via the memory of water effect (45). The non-local coherence of trillions of water molecules implied in the memory of water hypothesis amounts to entanglement at the molecular level. Thus, the intriguing possibility arises of describing the homeopathic process in terms of various 'layers' of entanglement, from the molecular up to the interactions of human beings (46). Thus, it is too early to give a definitive answer as to why some positive results are returned but a possible experimental test of non-local communication in clinical trials has been proposed (47). Now let us examine the effect on homeopathic provings of applying the DBRCT methodology of double-blinding and placebo control.

\section{DBRCTs of HPTs (aka Provings)}

The practice of homeopathy is founded on two pillars (48): repertories of prescribing symptoms and its extensive material medicae. In order for remedies to be included in a materia medica their clinical properties need to be discovered and this is done in 'provings', essentially HPTs. Here, an unknown remedy is given to healthy volunteers and the symptoms generated are recorded, collated and themes elucidated (49). Homeopaths then use this gathered information in a comparative manner (via repertorization after case-taking) to arrive at a suitable prescription for a patient during treatment.

From the point of view of remedies, it is interesting to assess treatment and proving scenarios in terms of information. Thus, homeopathic treatment involves use of remedy information by the practitioner after case taking. As previously stated, DBRCT methodology effectively breaks entanglement between patient, practitioner and remedy, in trials of individualized homeopathic prescribing. This could be brought 
about by the artificial blinding to the nature of the prescribed remedy that the homeopath and test subjects have to go through, leading to a loss of information. A proving, on the other hand, is a remedy information gathering exercise; the accumulated knowledge eventually becoming part of the materia medica.

Provings are not usually conducted in a double-blind placebo-controlled manner. After proving symptoms have been gathered, collation of the data allows a remedy picture to emerge. However, two DBRCTs of HPTs have recently been performed, and these showed that although there were clear differences in proving symptoms between remedy and placebo groups, there was also overlap or 'leakage' of symptoms between them $(50,51)$. These authors concluded that remedy and placebo groups must have become entangled. Interestingly, there has been some confirmation of this result recently by another research group (52). What this could mean is that in these proving DBRCTs, it is the loss of information (about what is remedy and what is placebo) that leads to entanglement formation.

This apparent contradiction between the DBRCT (entanglement-breaking) treatment and (entanglementcreating) proving situations is easily resolved when it is realized that entanglement between remedy and placebo is happening in both cases (however, in DBRCTs of homeopathic treatment, this leads to the breaking of entanglement between patient, practitioner and remedy). It suggests that it is not so much the effect of blinding on the making or breaking of entanglement that is important, rather the loss of information that blinding produces which is common to both. Making or breaking of entanglement can be seen therefore as epiphenomena of information loss brought on by blinding. This could be the key which permits the superposition of states that is the necessary condition for entanglement to occur. I shall return to this point later. Let us now consider the similarity between double-blind placebo-controlled provings and the famous double-slit experiment of quantum physics.

\section{The Double-Slit and Delayed Choice Experiments}

The double-slit experiment is one of those scientific classics $(53,54)$ as it has twice represented a turning point in our understanding of the fundamental particles of nature. The original experiment demonstrating the wave nature of light was performed by the 19th century scientist Thomas Young, and is shown in Fig. 1.

Thus, light is shone through a small hole in a screen, then onto another screen, this time with two closely-spaced holes. Light is diffracted through the two holes, spreads out and falls onto a third screen to form an interference pattern of light and shade (55).

The interference pattern can be best understood in terms of two overlapping sets of waves, as might be produced when two stones are dropped simultaneously into water.

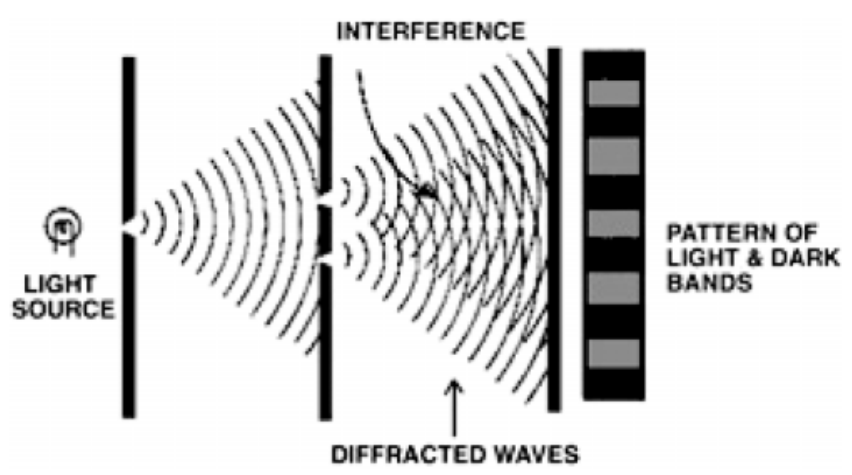

Figure 1. The double-slit experiment.

Where the two patterns meet and merge, wave peaks can interfere constructively (i.e. in-phase, they add together to produce an enhanced peak) or destructively (i.e. exactly $180^{\circ}$ out of phase, they cancel each other out). In the double-slit experiment, in-phase constructively interfering light waves produce a bright band, while out-of-phase destructively interfering light waves produce a dark band (see Fig. 1). However, other experiments, e.g. the photoelectric effect (54), demonstrate that light also behaves as a stream of particles, with each photon arriving in particulate fashion as a quantum, apparently independently of others. Thus, bizarrely, light seems to behave as waves or particles depending on the type of experiment that is performed.

Even more bizarrely, similar double-slit experiments can be performed on beams of particles, e.g. electrons, and even atoms and molecules (56). As particles, one would expect them to go through the slits and simply produce two patches of light with no interference pattern. However, a beam of electrons fired at a closely-spaced 'double-slit' (e.g. the serried ranks of atoms in a crystal: this acts as a three-dimensional diffraction grating - a series of slits-and is described by the same physics and physical principles as a double-slit) will produce an interference pattern of light and darkness on a scintillation screen placed down-range from the slits. This demonstrates that as far as traveling through the double-slit experiment is concerned, 'particle' electrons are behaving as 'waves'. But the fact that each electron produces a spot on a screen (even though the overall picture is one of an interference pattern) means that each electron is arriving as a particle. Under these circumstances, it might be thought possible to explain the interference pattern as a statistical effect of there being large numbers of particle-like electrons. This idea is dashed, however, when examining the results of firing electrons singly through the double-slit experiment, and the interference pattern still emerges.

Each electron individually goes through the experiment and strikes the screen to leave a spot. As spots build up, an interference pattern emerges. This means that although in lighting up the scintillation screen, each electron is behaving as a particle. It also means that by forming an interference pattern, each electron is behaving as a wave, and going through both slits at the same time. In effect, each electron is 
interfering with itself. However, a detector positioned after the slits to try to detect which one each electron might be going through [known as the delayed choice experiment (54)], causes the interference pattern on the screen to disappear. In its place are two clear spots, meaning that the electrons are now behaving as particles, when going through one or other of the slits, not both at the same time. Turning off the detector, re-establishes the interference pattern on the screen.

The same thing is noticed if electrons are replaced by photons of light or atoms or molecules. Thus, it is as if these quantum entities seem to 'know' in advance when and where they are being observed, the whole arrangement of the experiment, including its past and its future, and change their behavior accordingly. There is absolutely no way this extremely bizarre experimentally verified behavior can be explained according to the concepts of classical physics, and is why Nobel Laureate Professor Richard Feynman described the double-slit experiment as exemplifying 'the central mystery' of quantum theory $(53,54)$.

The double-slit and delayed choice experiments demonstrate the true meaning of particle-wave duality, which is that the whole experiment is an entangled entity: an entanglement which, according to the quantum theory of measurement, must include the states of the 'classical' (i.e. non-quantum) apparatus set up to observe the system (and the human beings making the observations: this is important to the analogy that will be developed later on because in this respect, the patient and practitioner may also be considered "classical pieces of apparatus' which nevertheless play host to the quantum states being considered). The behavior of any quantum entity in these experiments is affected by how we choose to observe it, but clearly the effect of this choice of observation is felt before it is made. Thus, from our everyday perspective, entangled quantum correlations appear not only to be able to operate instantaneously over space, but also over time itself (57).

\section{The Double-Slit Experiment and Double-Blind Placebo-Controlled Provings}

It is instructive to consider the double-slit experiment as an analogy to double-blind placebo-controlled provings. Thus, the blinding of remedy and placebo may be thought of as akin to the 'double-slit'. In just the same way as in the double-slit experiment it is unknown which hole an electron goes through (unless it is observed, the wave function governing its dynamics goes through both slits), so all involved in the proving experiment are ignorant of their prescription.

The entangled nature of the electron 'waves' going through the two slits is revealed by the interference pattern produced when they arrive at the scintillation screen. (In fact entanglement is usually used in a different sense, to express correlations between different entities. In the case of an individual electron going through both slits, entanglement is being used to express the superposition of the two components of the wave function representing the SAME entity.) Similarly, entanglement of remedy and placebo is revealed after observation of proving symptoms from human provers of the remedy/placebo, and the identity of remedy and placebo has been disclosed. Thus entanglement between the two groups of human provers (i.e. those on verum and those on placebo) may be thought of as an observed 'interference' pattern, produced by the double-blinding.

One way of formulating this situation [there are others, see $(23,44,45,58)]$ is to use the algebraic formalism of quantum theory. One has to bear in mind that human beings involved in clinical trials of homeopathy (and any other CAM therapy), or as provers in HPTs, are infinitely more complicated entities than sub-atomic particles! With that codicil very much in mind, in terms of wave functions, operators and expectation values used previously to describe PPR entanglement in the therapeutic process (22), an expression can be written relating a remedy/placebo proving symptom entangled state and a homeopathic observation operator, to a proving symptom expectation value:

$$
\left\langle\Psi_{\text {ent }}|\Pi o| \Psi_{\text {ent }}\right\rangle=\langle\Delta \boldsymbol{S x}\rangle
$$

where $\boldsymbol{\Psi}_{\text {ent }}$ represents the remedy/placebo groups' proving symptom entangled state wave function (for all the provers); חo is an operator representing the homeopathic coordinator(s)/ observer(s); and $\langle\boldsymbol{\Delta} \boldsymbol{S} \boldsymbol{x}\rangle$ represents the change in proving symptom expectation value (without at this stage defining any basis set for this expectation value). We may further define the remedy/placebo entangled state in terms of three-way PPR entanglement $(28,59)$ between all provers on the remedy $\mathbf{P}_{\mathbf{R x}}$, all provers on the placebo $\mathbf{P}_{\mathbf{P l}}$, and the homeopathic observers $\mathbf{P}_{\mathbf{O}}$ :

$$
\left.\left|\boldsymbol{\Psi}_{\text {ent }}\right\rangle=\frac{\mathbf{1}}{\sqrt{\mathbf{2}}}\left(\left|\mathbf{P}_{\mathbf{R x}} \uparrow \mathbf{P}_{\mathbf{P I}} \uparrow \mathbf{P}_{\mathbf{O}} \uparrow+\right| \mathbf{P}_{\mathbf{R X}} \downarrow \mathbf{P}_{\mathbf{P} \mathbf{l}} \downarrow \mathbf{P}_{\mathbf{O}} \downarrow\right\rangle\right)
$$

where $\mathbf{P}_{\mathbf{R x}} \uparrow$ and $\mathbf{P}_{\mathbf{R x}} \downarrow$ represent all provers on the remedy who may or may not show symptoms; $\mathbf{P}_{\mathbf{P} \uparrow} \uparrow$ and $\mathbf{P}_{\mathbf{P I}} \downarrow$ represent all provers on the placebo who may or not show symptoms; and $\mathbf{P}_{\mathbf{O}} \uparrow$ and $\mathbf{P}_{\mathbf{O}} \downarrow$ represent all proving observers who may or may not observe symptoms. As has been mentioned in earlier papers on PPR entanglement $(22,27,29)$, equations of type (8) represent just two of a total of eight maximally entangled states for three entangled entities. In terms of the double-slit simile, the eight entangled states [two of which are shown in Equation (8)] may be thought of as a superposition which appears as an 'interference pattern' of entangled state proving symptoms, which means $\mathbf{R}_{\mathbf{X}}$ and $\mathbf{R}_{\mathbf{P I}}$ cannot be distinguished.

\section{Breaking Entanglement: The Price of Information}

It is interesting to consider how disentanglement of remedy and placebo could be represented in terms of the double-slit metaphor. Thus, in the double-slit experiment, observation (i.e. gaining information) of electrons using a detector placed after the slits, 'collapses their wave function' (60) throughout 
the whole experiment, so that they leave their source and arrive at the screen as particles. This results in the interference pattern disappearing to be replaced by two clear spots.

The equivalent situation in the double-blind placebocontrolled proving is if provers and/or proving controllers/ observers know who has received the remedy and who has received placebo. Thus, remedy and placebo are no longer entangled (and the experiment can no longer be considered double-blinded).

In terms of the previous section's algebraic formulation, this would mean collapse of the entangled state wave function, so that $\Psi_{\text {ent }}=0$. This leaves the coordinators/observers in two quite separate two-way entangled relationships: with remedy provers and placebo provers. Thus:

- for the remedy:

$$
\left\langle\Psi_{\mathbf{R x}}\left|\Pi_{\mathbf{O}}\right| \Psi_{\mathbf{R x}}\right\rangle=\left\langle\Delta S_{R x}\right\rangle
$$

- and:

$$
\left|\Psi_{\mathbf{R x}}\right\rangle=\frac{1}{\sqrt{2}}\left(\left|\mathbf{P}_{\mathbf{R x}} \uparrow \mathbf{P}_{\mathbf{O}} \uparrow\right\rangle \pm\left|\mathbf{P}_{\mathbf{R x}} \downarrow \mathbf{P}_{\mathbf{O}} \downarrow\right\rangle\right)
$$

- for the placebo:

$$
\left\langle\Psi_{\mathbf{P I}}\left|\boldsymbol{\Pi}_{\mathbf{O}}\right| \boldsymbol{\Psi}_{\mathbf{P I}}\right\rangle=\left\langle\Delta S_{P l}\right\rangle
$$

- and:

$$
\left|\Psi_{\mathbf{P I}}\right\rangle=\frac{1}{\sqrt{2}}\left(\left|\mathbf{P}_{\mathbf{P I}} \uparrow \mathbf{P}_{\mathbf{O}} \uparrow\right\rangle \pm\left|\mathbf{P}_{\mathbf{P I}} \downarrow \mathbf{P}_{\mathbf{O}} \downarrow\right\rangle\right)
$$

In two-way entanglement, it turns out that there are four maximally entangled states (compared to eight for three entangled objects) so that Equations (10) and (12) each represent just two of these states (60). Also, as remedy and placebo are now disentangled, provers on placebo should not evince the symptoms shown by those on the remedy. Consequently in Equation (11), either $\left\langle\Delta S_{P l}\right\rangle=0$ or at least $\left\langle\Delta S_{P l}\right\rangle$ and $\left\langle\Delta S_{R x}\right\rangle$ cannot now be equated with each other. In other words, the act of removing blinding (i.e. providing information) should collapse the remedy/placebo entangled state wave function, leaving two completely distinguishable sets of results: one set for provers on the remedy, and one set for those on placebo. In terms of the double-slit metaphor, this would be analogous to the effect of observing the electrons before they strike the screen (i.e. loss of the interference pattern, which is replaced by two clearly defined spots).

The preceding discussion suggests a possible experimental test of this hypothesis. Thus, previous double-blind placebocontrolled provings should be repeated on a much larger sample of provers, but dividing the experiment into two halves so that blinded and unblinded protocols may be included. One half of the experiment would be double-blinded and placebocontrolled as before, while the other half could be selectively unblinded (e.g. either provers or coordinators) and used to observe how remedy and placebo might disentangle. From this perspective, the work of Schmidt et al. (47) in parapsychology might also provide some useful pointers toward experimental methodology.

\section{Quantum States as Representations of Knowledge}

Quantum information processing is one of the most interesting modern applications of quantum entanglement (4). Quantum computing, cryptography and teleportation are now all practically realizable technologies, at least in the laboratory (60). They are predicated on the realization that information is not independent of the quantum physical laws used to store and process it. Though quantum mechanics governs the way modern computers operate, the information they encode is still treated classically. The latest insights into the very foundations of quantum theory itself reveal that information is also subject to the same quantum laws (61).

Thus, in quantum teleportation experiments, information can completely and directly be transferred from one system to another, without that information traveling down any physically identifiable signaling pathway. The 'mechanism' which permits this direct transfer of information is quantum entanglement, and is completely different to any communication system developed so far. The entangled systems are more strongly coupled than classical systems, and together have well-defined informational characteristics. On their own, however, the individual systems may be completely random without any information content. Successful quantum teleportation means that the new teleported system becomes completely identical with the original, which by necessity has to disappear.

In 'classical' information theory, the elementary quantity of information is the bit which can have one of two values, e.g. 0 or 1 . Physical realization of a bit requires any system which can exist in two well-defined separate states. Where quantum information technology differs from previous 'classical' information technologies, is that a quantum system can be in a superposition of both the 0 and 1 states (called a qubit). There is no parallel in classical information theory. Far from superposition leading to a loss of information, it offers a completely different way of encoding information onto two or more qubits which actually uses the entangled superposition of states. The information is encoded in such a way that neither of the two qubits carries any well-defined information on its own: all of the information is encoded in the joint properties of their entangled state. Any attempt to 'get at' the presumed information content of the individual qubits is doomed because it means breaking the entanglement between them, leading to the loss of information.

Quantum teleportation raises profound issues about the nature of reality, especially at the quantum level. Thus, information or knowledge of a system can have a more fundamental meaning than the system's objective reality or, to put it even more starkly, we can only concern ourselves 
with what can be known about the reality of a system; not its presumed objective (ontological) 'reality-in-itself'. Measurement (observation) is what changes a quantum state but it is in investing this state with too much 'reality' (as an object 'out there', independent of our observation of it) that leads to the well-known paradoxes of quantum theory, such as Schrödinger's Cat $(53,54)$. These conundra disappear if the quantum state of a system is taken as a representation of knowledge (what we can know) about it, not its presumed objective 'reality in itself'.

Although quantum teleportation works via entangled states over a distance, it is also necessary to access a completely separate and classical code in order to make any sense of the encoding of information in the entangled states. This is an additional requirement for the usage of information transfer via entangled states, and explains the difference between quantum information and classical information. However, this raises the problem that entangled states in themselves cannot be treated as if they could be the source of causal signals in the classical sense: if treated as such, the entangled state is broken and the information lost. [The explanation of this state of affairs is a sophisticated argument which rests on the assumption that the standard linear formalism of quantum mechanics forbids the use of entanglement for superluminal (i.e. faster than light or backwards in time) communication] (57).

Now, by analogy with quantum teleportation and quantum encryption, clinical trials are by their very nature classical 'detectors' of causality. Consequently, they tend to regard treatment groups as similar to causal signals, which can be distilled out of sequences of clinical trials. This, it has been argued, is one of the reasons why clinical trials cannot be used for a detection of effects that are based on non-local mechanisms: they lead to loss of information of entangled states when 'intercepted' as causal signals $(62,63)$.

The thrust of this and earlier papers in this series is that it should be possible to use notions of quantum entanglement (and by implication, information processing) to illustrate certain features of the therapeutic process in homeopathy and other CAMs. Consequently, the effects of investigating homeopathy and other CAMs using blinded trial procedures should also be amenable to such illustration. Thus, in doubleblinded provings $(50,51)$, each of the components in the PPR entangled state may be thought of as two-state 'macro-qubits' (e.g. Pr can be helpful or unhelpful; Px can be well or unwell; and $\mathrm{Rx}$ can be curative or non-curative) and, therefore, by implication, the homeopathic process might be considered to involve macro-quantum 'teleportation'. In this respect, the idea of 'macro-quantum teleportation' would have certain similarities to Walach's notion of generalized entanglement, based on semiotics $(58,63,64)$. However, it is only the entangled state which contains information about the whole system. Thus, anything which breaks the entangled state will necessarily lead to loss of information about the integration of function of the systems as a whole system. Clearly, this could happen in DBRCTs of homeopathic efficacy, where either the remedy or patient and practitioner are removed from their entangled therapeutic context $(18,27)$. In the double-blind proving situations, although information appears to be lost about the individual remedy and placebo, the resulting entanglement provides information about the whole experimental situation.

\section{Conclusion}

Though still a controversial assertion, macroentanglement could be a necessary concomitant of the homeopathic (indeed many CAMs') therapeutic process. Thus, the breaking of macroentanglement (in this case, between patient, practitioner and remedy) could explain the failure of DBRCTs to provide unequivocal evidence for or against the efficacy of homeopathy (18). Conversely, entanglement formation could explain the inability of DBRCTs of HPTs to distinguish between proving and placebo groups.

This apparent contradiction between DBRCTs of homeopathic efficacy and homeopathic provings was explained in terms of information loss brought about by quantum superposition, i.e. entanglement formation, between the quantum wave functions of remedy and placebo by the double-blinding protocol. In the proving situation, similarities with the famous double-slit experiment of quantum physics, and quantum information processing are proposed.

Thus, in the double-slit experiment, the wave nature of quantum particles is demonstrated by their formation of an interference pattern on a screen. However, attempts to make observations of this situation at one or other slit prior to the waves arriving at the screen destroys the superposition, leading to their behaving as particles. This demonstrates that superposition is the key to their entanglement. In quantum information processing, any attempt to isolate entangled 'qubits' leads to collapse of the entangled state and the loss of information.

Therefore, an algebraic argument has been proposed that if remedy and placebo really are entangled by application of the DBRCT methodology to homeopathic provings, then any attempt to have prior knowledge of them both should break the entangled proving state. This should then deliver results showing clear differences between remedy and placebo in terms of proving symptoms. Thus, the use of DBRCTs for testing homeopathy would appear to be a flawed strategy as they seem to destroy the very effects they were purportedly designed to investigate. Clearly, such a rationale could be applied to many CAMs and perhaps even to DBRCTs of conventional medicine. For as Nelson intimated, when one looks at the world with a blind eye, one tends not to see anything.

\section{Acknowledgments}

I wish to express my warm thanks and appreciation to Ms Clare Relton RSHom, MSc, from The Society of Homeopaths, UK, for help in preparing this manuscript. 


\section{References}

1. The Oxford Dictionary of Quotations, 3rd edition. London: Book Club Association, 1981, 360

2. Ernst E. Equivalence and non-inferiority trials of CAM. Evid Based Complement Alternat Med 2004;1:9-10.

3. Milgrom LR. Entanglement, knowledge, and there effects on the outcomes of blinded trials of homeopathic provings. J Altern Complement Med 2006;12:271-9.

4. Zeilinger A. Fundamentals of quantum information. Phys World March (online) 1998. Available at: http: //physicsweb.org/articles/world/ $11 / 3 / 9$.

5. Lewith G, Jonas WB, and Walach H. In: Clinical Research in Complementary Therapies: Principles, Problems, and Solutions, London: Churchill-Livingston, 2003.

6. Shang A, Huwiler-Müntener K, Nartey L, Juni P, Dorig S, Sterne LA, et al. Are the clinical effects of homeopathy placebo effects? Comparative study of placebo-controlled trials of homeopathy and allopathy. Lancet 2005;366:726-32.

7. Vandenbrouke JP. Homeopathy and the 'growth of truth'. Lancet 2005;366:691-2.

8. Fisher P. Homeopathy and The Lancet. Evid Based Complement Alternat Med 2006;3:145-7.

9. Bell IR. All evidence is equal, but some evidence is more equal than others: can logic prevail over emotion in the homeopathy debate? J Altern Complement Med 2005;11:763-9.

10. Frass M, Schuster E, Muchitsch I, Duncan J, Gei W, Kozel G, et al. Bias in the trial and reporting of trials of homeopathy: a fundamental breakdown in peer review and standards?. J Altern Complement Med 2005;11:780-2.

11. Kienle H. Failure to exclude false negative bias: a fundamental flaw in the trial of Shang et al. J Altern Complement Med 2005;11:783.

12. Peters D. Shang et al. carelessness, collusion, or conspiracy? J Altern Complement Med 2005;11:779-80.

13. Murcott T. The Whole Story: Alternative Medicine on Trial? New York: Macmillan, 2005.

14. Mathie RT. The research evidence base for homeopathy: a fresh assessment of the literature. Homeopathy 2003;92:84-91.

15. Van Wassenhoven M. Priorities and methods for developing the evidence profile of homeopathy: recommendations of the $\mathrm{ECH}$ General Assembly and XVIII Symposium of GIRI. Homeopathy 2005;94:107-24.

16. Bellavite P, Ortolani R, Pontorollo F, Piasere V, Benato G, Conforti A. Immunology and homeopathy. 4. Clinical studies-Part 2. Evid Based Complement Alternat Med 2006, Advanced access, 31/07/2006; doi: 10.1093/ecam/nel046, and references therein.

17. Bellavite P, Ortolani R, Pontorollo F, Piasere V, Benato G, Conforti A. Immunology and homeopathy. 4. Clinical studies-Part 1. Evid Based Complement Alternat Med 2006;3:293-301, and references therein.

18. Weatherley-Jones E, Thompson EA, Thomas KJ. The placebo-controlled trial as a test of complementary and alternative medicine: observations from research experience of individualised homeopathic treatment. Homeopathy 2004;93:186-9.

19. Cramer JG. Quantum Non-Locality and the Possibility of Superluminal Effects. Proceedings of the NASA Breakthrough Propulsion Physics Workshop, Cleveland, OH, August, 1997.

20. Aspect A, Granger P, Roger R, et al. Experimental realisation of Einstein-Podolsky-Rosen-Bohm gedanken [thought] experiment: a new violation of Bell's inequalities. Phys Rev Lett 1982;49:91-4.

21. Atmanspacher H, Römer H, Walach H. Weak quantum theory: complementarity and entanglement in physics and beyond. Found Phys 2002;32: 379-406.

22. Milgrom LR. Patient-practitioner-remedy (PPR) entanglement: Part 4: Towards classification and unification of the different entanglement models for homeopathy. Homeopathy 2004;93:34-42.

23. Hyland ME. Does a form of "entanglement" between people explain healing? An examination of hypotheses and methodology. Complement Ther Med 2004;12:198-208.

24. Gernert D. Conditions for entanglement. Front Perspect 2005;14:8-13.

25. Grinberg-Zylberbaum J, Delaflor M, Attle L, Goswami A. The EinsteinPodolsky-Rosen paradox in the brain: the transferred potential. Phys Essays 1994;7:423-8.

26. Wackermann J. Dyadic correlations between brain functional states: present and future perspectives. Mind Matter 2004;2:105-22.
27. Milgrom LR. Are randomised controlled trials (RCTs) redundant for testing the efficacy of homeopathy? A critique of RCT methodology from the theoretical standpoint of patient-practitioner-remedy (PPR) entanglement. J Altern Complement Med 2005;11:831-8.

28. Greenberger DM, Horne MA, Shimony A, Zeilinger A. Bell's theorem without inequalities. Am J Phys 1990;58:1131-43.

29. Milgrom LR. Patient-practitioner-remedy (PPR) entanglement. Part 8: 'Laser-like' action of the homeopathic therapeutic encounter as predicted by a gyroscopic metaphor for the vital force. Forsch Komplementarmed Klass Naturheilkd 2005;12:206-13.

30. Bignamini M, Saruggia M, Sansonetti G. Homeopathic treatment of anal fissures using Nitricum acidicum. Berl J Res Hom 1991;1:286-7.

31. Labrecque M, Audet D, Latulippe LG, Drouin J. Homeopathic treatment of plantar warts. Can Med Assoc J 1992;146:1749-53.

32. Leaman AM, Gorman D. Cantharis in the early treatment of minor burns. Arch Emerg Med 1989;6:259-61.

33. Andrade L, Ferraz MB, Atra E, Castro A, Silva MS. A randomised controlled trial to evaluate the effectiveness of homeopathy in rheumatoid arthritis. Scand J Rheumatol 1991;20:204-8.

34. Chapman EH, Angelica J, Spitalny G, Strauss M. Results of a study of the homeopathic treatment of PMS. J Am Inst Hom 1997;87:14-21.

35. Gibson RG, Gibson S, MacNeill AD, Watson-Buchanan W. Homeopathic therapy in rheumatoid arthritis: evaluation by double-blind clinical therapeutic trial. Br J Clin Pharmacol 1980;9:453-9.

36. Jacobs J, Jimenez LM, Gloyd SS. Treatment of acute childhood diarrhea with homeopathic medicine: A randomized clinical trial in Nicaragua. Pediatrics 1994;93:719-25.

37. Kent JT. Lectures on Homeopathic Philosophy. Lecture XXXVI: The Second Prescription, Sittingbourne, UK: Homeopathic Book Service, 1990.

38. Schiff M. The Memory of Water: Homeopathy and the Battle of Ideas in the New Science, London: Thorsons (Harper-Collins), 1995.

39. Hankey A. A Maddox effect? A reason to adopt time series protocols in tests of homeopathic remedies. J Altern Complement Med 2005;11: 759-61.

40. Bohr N. Can a quantum mechanical description of physical reality be considered complete? Phys Rev 1935;48:609-702.

41. Schmid GB. Much ado about entanglement: a novel approach to test non-local communication via violation of local realism. Forsch Komplementarmed Klass Naturheilkd 2005;12:206-13.

42. Vallance AK. Can biological activity be maintained at ultra-high dilution? An overview of homeopathy, evidence, and Bayesian philosophy. J Altern Complement Med 1998;4:49-76.

43. Weingärtner O. An approach to the scientific identification of a therapeutically active ingredient of high potencies. Forsche Komplementarmed Klass Naturhelkd 2002;9:229-33.

44. Hyland ME. Entanglement and some heretical thoughts about homeopathy. Homeopathy 2004;94:105-6.

45. Del Guidice E, Preparata G, Vitiello G. Water as a free electron dipole laser. Phys Rev Lett 1988;61:1085-8.

46. Milgrom LR. The sound of two hands clapping: could homeopathy work locally and non-locally? Homeopathy 2005;94:100-4.

47. Schmidt H, Morris R, Rudolph L. Channeling evidence for a psychokinetic effect on independent observers. J Parapsychol 1986;50:1-15.

48. Walach H. The pillar of homeopathy: remedy provings in a scientific framework. Br Hom J 1997;86:219-24.

49. Sherr J. The Dynamics and Methodology of Homeopathic Provings, West Malvern, UK: Dynamis Books, 1994.

50. Möllinger H, Schneider R, Löffel M, et al. A double blind randomized homeopathic pathogenic trial with healthy persons: comparing two high potencies. Forsche Komplementarmed Klass Naturheilkd 2004;11: 274-80.

51. Walach H, Sherr J, Schneider R, Shabi R, Bond A, Rieberer G. Homeopathic proving symptoms: result of a local, non-local, or placebo process? A blinded, placebo-controlled pilot study. Homeopathy 2004;93: 179-85.

52. Dominici G, Bellavite P, di Stanislao C, Gulia P, Pitari G. Double-blind placebo-controlled homeopathic pathogenic trials: symptom collection and analysis. Homeopathy 2006;95:123-30.

53. Gribbon J. Schrödinger's kittens and the search for reality. London: Phoenix, 1995

54. Gribbon J. $Q$ is for quantum. London: Weidenfeld and Nicholson, 1998.

55. Pain HJ. The Physics of Vibrations and Waves, 5th edition, Chichester, UK: John Wiley and Son, 2002. 
56. Zeilinger A. Quantum experiments and the foundations of physics. Talk given to the Brookhaven National Laboratory, February 28, 2001.

57. Cramer JG. The transactional interpretation of quantum mechanics. Rev Mod Phys 1986;58:647-87.

58. Walach $\mathrm{H}$. Entanglement model of homeopathy as an example of generalised entanglement predicted by weak quantum theory. Forsche Komplementarmed Klass Naturheilkd 2003;10:192-200.

59. Milgrom LR. Patient-practitioner-remedy (PPR) entanglement. Part 5: Can homeopathic remedy reactions be outcomes of PPR entanglement? Homeopathy 2004;93:94-8.

60. Wooters WK. Quantum entanglement as a quantifiable resource. Philos Trans $R$ Soc London A 1998:356:1717.
61. Zeilinger A. Quantum teleportation and the nature of reality. 2004, Available at: http: //www.btgjapan.org/catalysts/anton.html.

62. Walach H. Entangled - and tied in knots! Practical consequences of an entanglement model for homeopathic research and practice. Homeopathy 2005;94:96-9.

63. Walach H. Generalised entanglement: a new theoretical model for understanding the effects of complementary and alternative medicine. J Altern Complement Med 2005;11:549-59.

64. Walach $\mathrm{H}$. Magic of signs: a non-local interpretation of homeopathy. Br Hom J 2000;89:127-40.

Received July 8, 2006; accepted August 15, 2006 


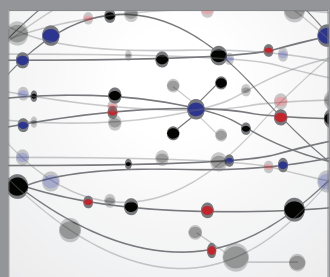

The Scientific World Journal
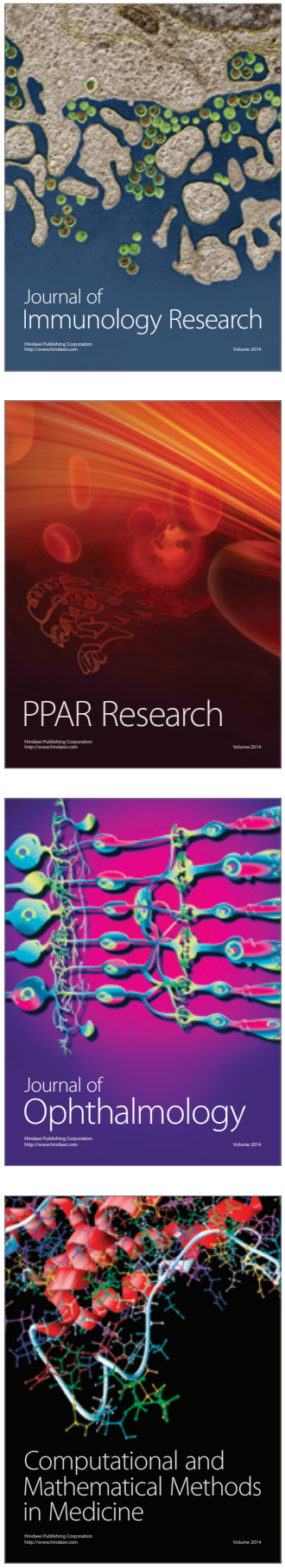

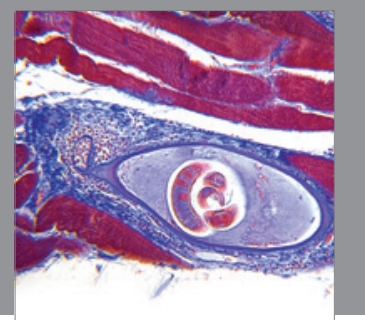

Gastroenterology

Research and Practice


\section{Hindawi}

Submit your manuscripts at

http://www.hindawi.com
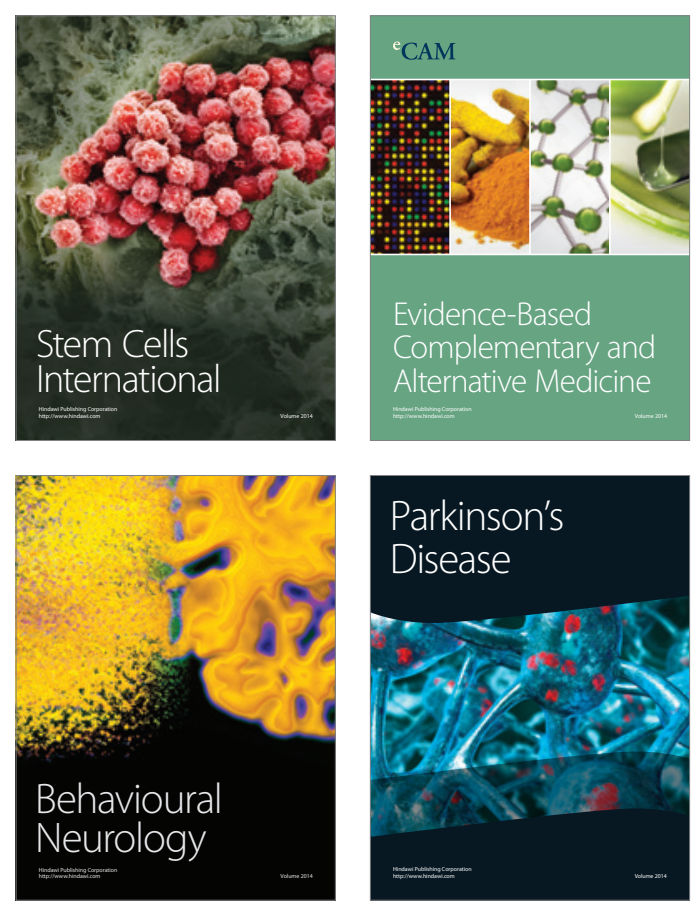

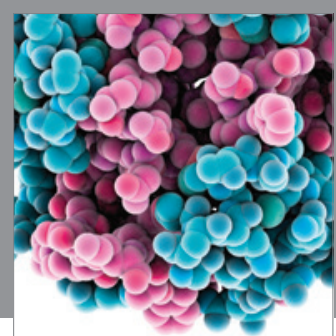

Journal of
Diabetes Research

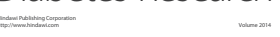

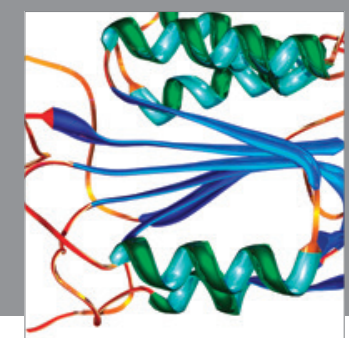

Disease Markers
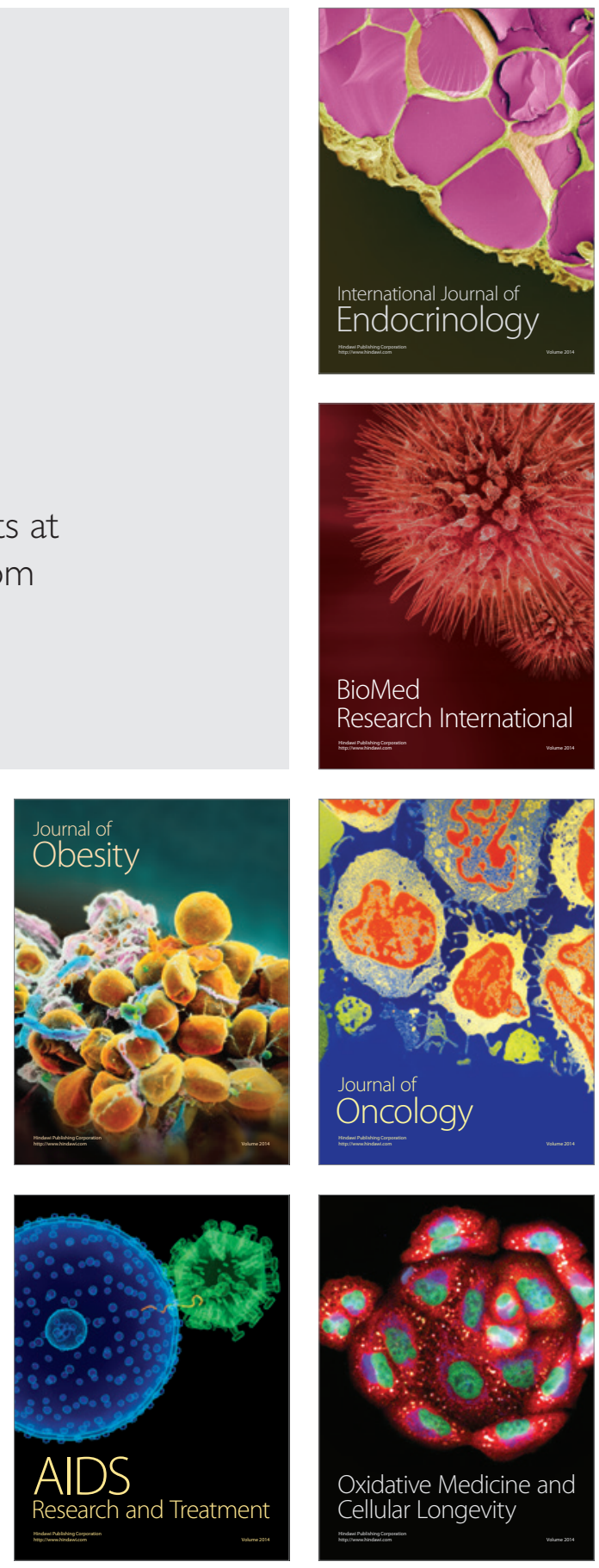